\title{
Direct comparisons between VERA, VLBA and EVN astrometry results and an analytic gas dynamics model
}

\author{
Nobuyuki Sakai ${ }^{* 1}$ \\ ${ }^{1}$ Mizusawa VLBI Observatory, National Astronomical Observatory of Japan, Mitaka, Tokyo \\ 181-8588 \\ E-mail: nobuyuki.sakai@nao.ac.jp
}

Context. The Perseus arm, the nearest main arm from the Sun, has been studied by multiwavelength observations and numerical simulations. It has been revealed that the Perseus arm has an effect on gas kinematics known as the streaming motion (i.e., systematic peculiar motion), while direct comparisons between the observations and the simulations have been limited due to insufficient spatial/velocity resolutions.

Aims. We aim to model the peculiar motion, and to derive physical parameters of the Perseus arm. Using the parameters, we discuss the role of the main arm in the Milky Way.

Methods. We directly compare VLBI astrometry results for the Perseus arm, including accurate $3 \mathrm{D}$ position and velocity information, with the analytic gas dynamics model proposed by PiñolFerrer et al. (2012).

Results. We succeeded to explain the VLBI astrometry results with seven model parameters. The model showed an offset between a dense gas region and the bottom of the spiral potential model. Conclusion. Astrometry results can be used to judge previous theoretical works explaining the nature of the spiral arm (e.g., the density-wave theory, the recurrent transient spiral, etc.). The offset predicted from our model can be confirmed by stellar astrometry (e.g., Gaia astrometry). A combination of gas and stellar astrometry results will drastically change our understanding of the spiral arm in the near future.

12th European VLBI Network Symposium and Users Meeting,

7-10 October 2014

Cagliari, Italy

\footnotetext{
* Speaker.
} 


\section{Introduction}

In 1964, the density-wave theory was proposed by Lin \& Shu (1964) to overcome the winding dilemma, in which a spiral arm in a disk galaxy is destroyed within a time scale of several galactic rotations due to the differential rotation. After that, the density-wave theory has been used to discuss the evolution and dynamics of a spiral arm in a disk galaxy. For instance, Fujimoto (1968) and Roberts (1969) examined non-linear gas motion perturbed by the density wave (the spiral arm) numerically, and found the "Galactic shock" where velocity jump (non-circular motion) occurred and the gas accumulated behind the bottom of the spiral potential (see fig. 5 in Roberts 1969).

Ever since 1960s, the density-wave theory has been supported by simulations and observations. However, the previous observations were mainly based on line-of-sight velocities (1D information) and 2D positions on the celestial sphere, which were not enough to conduct quantitative comparisons between the observations and the simulations in terms of both velocity and spatial resolutions.

Since 2000s Very Long Baseline Interferometry (VLBI) technique has allowed us to conduct Galactic-scale astrometry and to obtain accurate information about 3D positions and velocities of Galactic masers in the Milky Way (e.g., Reid \& Honma 2014a; Reid et al. 2014b). At the moment, we can conduct the direct (quantitative) comparisons between the astrometry results and Galactic dynamical models for understanding the dynamics and evolution of the spiral arm, as well as those of the Milky Way.

For the first step we compare between VLBI astrometry results and a gas dynamics model based on the density-wave theory in this paper.

\section{Method:The least-squares fits}

Table 1: Summary of the spiral potential parameters used in this paper

\begin{tabular}{cll}
\hline Parameter & Dimension & Notes \\
\hline$\sqrt{\Phi_{\mathrm{a}}}$ & $\left(\mathrm{km} \mathrm{s}^{-1}\right)$ & Amplitude of the spiral potential \\
$R_{\mathrm{ref}}$ & $(\mathrm{kpc})$ & Reference position of the spiral potential \\
$i$ & $(\mathrm{deg})$ & Pitch angle of the spiral potential \\
$\Omega_{\mathrm{p}}$ & $\left(\mathrm{km} \mathrm{s}^{-1} \mathrm{kpc}^{-1}\right)$ & Pattern speed of the spiral potential \\
$m$ & & Mode of the spiral potential \\
$\lambda$ & $\left(\mathrm{km} \mathrm{s}^{-1} \mathrm{kpc}^{-1}\right)$ & Damping term for gas motion \\
$\varepsilon$ & $\left(\mathrm{km} \mathrm{s}^{-1} \mathrm{kpc}^{-1}\right)$ & Co-rotation softening parameter \\
\hline
\end{tabular}

For comparisons between the analytic gas dynamics model (Pinõl-Ferrer et al. 2012) and previous VLBI astrometry results (e.g., Reid et al. 2014b), we searched the minimum value of the reduced chi-square $\left(\chi_{v}^{2}\right)$ in the analytic model parameters which were varied within appropriate ranges. The chi-square $\left(\chi^{2}\right)$ can be simply written as

$$
\chi^{2}=\sum_{i=1}^{n}\left[\frac{\Delta a_{i}}{\sigma_{i}}\right]^{2},
$$


where $a_{i}$ is a measurement with an uncertainty $\sigma_{i}$, and $\Delta a_{i}$ is the residual between the measurement and an expected value. We used peculiar motions ( $U$ and $V$ ) with uncertainties referred from Reid et al. (2014b) for the measurements, and the residuals were calculated using the measurements and the analytic model.

Table 1 displays the analytic model parameters used for a spiral potential, while we assumed a flat rotation model (i.e., $\left.\Theta(R)=\Theta_{0}\right)$ and Galactic constants of $\left(R_{0}, \Theta_{0}\right)=(8.33 \mathrm{kpc}, 240 \mathrm{~km}$ $\mathrm{s}^{-1}$ ) to describe a Galactic axisymmetric potential. Note that $\Theta(R)$ shows the rotation speed at a Galactocentric distance $R$. There are seven parameters of which three parameters, the amplitude, pitch angle, and mode of the spiral potential, were fixed based on previous researches.

For the amplitude of the spiral potential, we referred to Grosbøl et al. (2004) who showed relation between the pitch angle and the amplitude for disk (spiral) galaxies (see fig. 8 in Grosbøl et al. 2004). Spiral amplitudes ranged between $\sim 0$ and $\sim 50 \%$ relative to disk amplitudes in Grosbøl et al. (2004), and therefore we fixed the amplitude between 10 and $50 \%$ relative to the local amplitude with an increment of $10 \%$. Note that the amplitude can be calculated by eq. (6.30) in Binney \& Tremaine (2008). Surface density $\Sigma$ is related to the amplitude and a local surface density $\Sigma_{\odot}$ of $63.9 \mathrm{M}_{\odot} \mathrm{pc}^{-2}$ was assumed based on Mcmillan (2011).

For the pitch angle and mode of the spiral arm, we cited Vallée (2014) who listed recent results of spiral arms in the Milky Way. Pitch angles between 2.3 (or 5.4) and 16.5 deg were listed with a mode of 2 or 4 in tables 1 and 2 of Vallée (2014). Note that the pitch angle of 2.3 deg was determined based on only two sources associated the Outer arm in Reid et al. (2009), and recently the value was modified to $13.8 \pm 3.3 \mathrm{deg}$ based on six sources associated with the Outer arm in Reid et al. (2014b). Thus, we fixed the pitch angle between 5 and 20 deg with an increment of 5 deg. Also, we fixed the mode to be 2 or 4 .

Aside from the fixed values, we may not have to fix the reference position of the spiral potential $\left(R_{\text {ref }}\right)$ based on VLBI astrometry results, since the VLBI astrometry results may not trace the bottom of the spiral potential. Therefore, we searched it with an increment of $0.1 \mathrm{kpc}$ in a range corresponding to phase between $-\pi$ and $+\pi$ radian of the spiral potential from $R_{\text {ref }}=10.4 \mathrm{kpc}$ determined by VLBI astrometry. Note that the search range of $R_{\text {ref }}$ depends on not only the pitch angle, but also the mode of the spiral potential. For instance, in the case of $\mathrm{i}=5.0^{\circ}$ and $\mathrm{m}=4$, we searched $R_{\text {ref }}$ between 9.6 and $11.0 \mathrm{kpc}$, while we searched $R_{\text {ref }}$ between 5.9 and $18.4 \mathrm{kpc}$ in the case of $\mathrm{i}=20.0^{\circ}$ and $\mathrm{m}=2$.

For the pattern speed of the spiral potential $\left(\Omega_{p}\right)$, we searched it between 10 and $30 \mathrm{~km} \mathrm{~s}^{-1}$ $\mathrm{kpc}^{-1}$ with an increment of $1 \mathrm{~km} \mathrm{~s}^{-1} \mathrm{kpc}^{-1}$ based on previous researches (e.g., $\Omega_{p}=11.5 \pm 1.5$ $\mathrm{km} \mathrm{s}^{-1} \mathrm{kpc}^{-1}$ in Gordon 1978; $\Omega_{p}=30 \mathrm{~km} \mathrm{~s}^{-1} \mathrm{kpc}^{-1}$ in Fernández et al. 2001).

The other two parameters $(\lambda$ and $\varepsilon$ ) are not basically observables, and therefore we searched them in a relatively wide range. $\lambda$ and $\varepsilon$ were searched in the same range between 1 and $30 \mathrm{~km} \mathrm{~s}^{-1}$ $\mathrm{kpc}^{-1}$ with an increment of $1 \mathrm{~km} \mathrm{~s}^{-1} \mathrm{kpc}^{-1}$.

After the grid (discrete) search, we determined final values with errors using the formula provided by Bevington \& Robinson (2002) as

$$
\chi^{2}=\frac{\left(a_{j}-a_{j}^{\prime}\right)^{2}}{\sigma_{j}^{2}}+C,
$$


where $a_{j}$ is a single parameter in the vicinity of the minimum (at $a_{j}^{\prime}$ ) of the $\chi^{2}$ distribution, $\sigma_{j}^{2}$ is a dispersion of the single parameter, and the constant $C$ is a function of the uncertainties $\sigma_{i}$ (as explained in eq. 2.1) and the other parameters $a_{k}$ for $k \neq j$.

However, we note that the error obtained from eq. 2.2 might be as a guide, since systematic error could be dominant in the spiral potential model.

\section{Results \& Discussion}

Table 2: Results of the least-squares fits for the spiral potential parameters*

\begin{tabular}{|c|c|c|c|c|c|c|c|c|c|c|}
\hline $\begin{array}{l}\text { Model } \\
\text { ID }\end{array}$ & $\begin{array}{c}\text { \# of } \\
\text { sources }\end{array}$ & $\sqrt{\Phi_{\mathrm{a}}}{ }^{\dagger}$ & $R_{\text {ref }}$ & $i$ & $\Omega_{p}$ & $m$ & $\lambda$ & $\varepsilon$ & $\chi^{2} /$ d.o.f & $\mathrm{Memo}^{\dagger}$ \\
\hline 1 & 27 & 15.3 & $9.46 \pm 0.01$ & 5.0 & $24.1 \pm 0.1$ & 2 & $27.0 \pm 2.5$ & $0.99 \pm 0.03$ & $549 / 50$ & $30 \%$ of $\Sigma_{\odot}$ \\
\hline 2 & 27 & 28.1 & $9.39 \pm 0.02$ & 10.0 & $17.8 \pm 0.8$ & 2 & $24.4 \pm 1.9$ & $3.2 \pm 0.2$ & $339 / 50$ & $50 \%$ of $\Sigma_{\odot}$ \\
\hline 3 & 27 & 34.7 & $9.10 \pm 0.04$ & 15.0 & $17.9 \pm 0.4$ & 2 & $18.9 \pm 1.7$ & $4.2 \pm 0.1$ & $321 / 50$ & $50 \%$ of $\Sigma_{\odot}$ \\
\hline 4 & 27 & 36.2 & $9.16 \pm 0.05$ & 20.0 & $10.1 \pm 0.2$ & 2 & $6.1 \pm 0.3$ & $13.2 \pm 0.3$ & $319 / 50$ & $40 \%$ of $\Sigma_{\odot}$ \\
\hline 5 & 27 & 12.5 & $9.74 \pm 0.01$ & 5.0 & $24.1 \pm 0.1$ & 4 & $7.7 \pm 0.6$ & $0.98 \pm 0.03$ & $684 / 50$ & $40 \%$ of $\Sigma_{\odot}$ \\
\hline 6 & 27 & 19.8 & $9.22 \pm 0.01$ & 10.0 & $10.1 \pm 0.3$ & 4 & $1.7 \pm 0.2$ & $8.8 \pm 0.3$ & $446 / 50$ & $50 \%$ of $\Sigma_{\odot}$ \\
\hline 7 & 27 & 24.6 & $9.01 \pm 0.02$ & 15.0 & $11.5 \pm 0.3$ & 4 & $5.5 \pm 0.3$ & $9.3 \pm 0.3$ & $349 / 50$ & $50 \%$ of $\Sigma_{\odot}$ \\
\hline 8 & 27 & 25.6 & $9.48 \pm 0.03$ & 20.0 & $16.0 \pm 0.1$ & 4 & $7.0 \pm 0.3$ & $7.0 \pm 0.2$ & $306 / 50$ & $40 \%$ of $\Sigma_{\odot}$ \\
\hline
\end{tabular}

$*$ Bold values, $\sqrt{\Phi_{\mathrm{a}}}, i$, and $m$, were fixed in the least-squares fits (see text). The other parameters, $R_{\mathrm{ref}}, \Omega_{\mathbf{p}}, \lambda$, and $\varepsilon$, were searched within realistic values in the least-squares fits (see also text).

$\dagger \sqrt{\Phi_{\mathrm{a}}}$ is proportional to surface density $\Sigma$ (see eq. 6.30 in Binney \& Tremaine 2008). $\sqrt{\Phi_{\mathrm{a}}}$ was converted into the local surface density $\Sigma_{\odot}$, and the value is listed in the last column (see text).

Through the least-squares fits described in the previous chapter, we determined the reduced $\chi^{2}$ values $\left(\chi_{v}^{2}\right)$ in various combinations of the model parameters as listed in table 2 . From model ID-1 to ID-4, the mode $m=2$ was fixed. The models ID-3 and ID-4 (in the cases of $i=15.0^{\circ}$ and $20.0^{\circ}$ ) showed lower $\chi_{v}^{2}$ values compared to ID-1 and ID-2. From ID-5 to ID-8, the mode $m=4$ was fixed, and ID- 8 (in the case of $\mathrm{i}=20.0^{\circ}$ ) showed the minimum $\chi_{v}^{2}$ value in the four models.

Based on the all $\chi_{v}^{2}$ values, we regard the model ID- 8 as a good model in this paper. We note that this result is the first step result, since we have to sophisticate both the fitting procedure (i.e., the least-squares fit) and the analytic model. For instance, we assumed constant pitch angle and amplitude for the spiral potential, which may not represent the real Galaxy (e.g., bifurcation and spur of the spiral arm). However, the sophistication of the model is beyond the scope of this paper.

Figure 1 displays gas orbits model generated from ID-8. Observed and modeled peculiar motions for the Perseus arm are superimposed on the gas orbits model (see fig. 1), and also amplitude of the spiral potential (ID-8) is represented in the figure. Interestingly, the gas orbits of ID-8 concentrate around the sources located in the Perseus arm, meaning that our model (ID-8) based on the observed peculiar motions can predict the dense gas region precisely. The dense gas region is located at outer edge of the spiral potential (see fig. 1), which is consistent with Pinõl-Ferrer et al. (2012), but not consistent with the galactic shock proposed by Fujimoto (1968) and Roberts (1969). The difference of the gas distribution may originate in difference between numerical (Fujimoto 1968; Roberts 1969) and analytic (Pinõl-Ferrer et al. 2012) solutions. 


\section{Conclusion}

We succeeded to conduct direct (quantitative) comparison between VLBI astrometry results and an analytic gas dynamics model for testing the density-wave theory, while the direct comparison has suffered from observational limitations such as insufficient spatial and velocity resolutions since 1964, in which the density-wave theory was proposed by Lin \& Shu (1964). We obtained some indications from the direct comparison, although sophistication of the model should be conducted (e.g., bifurcation of spiral arm).

For instance, our model suggested different pitch angles of gas and (probably) stellar spiral arms. Pitch angle of the Perseus arm was obtained to be $9.9 \pm 1.5$ deg by VLBI astrometry results in Choi et al. (2014), while that of the spiral potential model was obtained to be $\sim 20 \mathrm{deg}$. The difference of the pitch angles will be able to be judged by stellar astrometry (e.g., Gaia astrometry). In addition, dense gas regions were deviated from bottom of the spiral potential model (see fig. 1), which will be also judged by stellar astrometry.

In the Gaia era, a combination of gas and stellar astrometry would be a powerful tool to discriminate several dynamics models such as the density-wave theory and the recurrent transient spiral proposed by mainly numerical simulations (e.g., Miller et al. 1970). The model selection will allow us to understand the origin and evolution of the spiral arm, as well as those of the Milky Way.

\section{References}

[1] Bevington, P. R., \& Robinson, D. K. 2002, Data Reduction and Error Analysis for the Physical Sciences, (3rd ed.; New York, NY: McGraw-Hill)

[2] Binney, J., \& Tremaine, S. 2008, Galactic dynamics, (2nd ed.; Princeton, NJ: Princeton Univ. Press)

[3] Choi, Y. K., Hachisuka, K., Reid, M. J., et al. 2014, ApJ, 790, 99

[4] Fernández, D., Figueras, F., \& Torra, J. 2001, A\&A, 372, 833

[5] Fujimoto, M. 1968, IAUS, 29, 453

[6] Gordon, M. A. 1978, ApJ, 222, 100

[7] Grosbøl, P., Patsis, P. A., \& Pompei, E. 2004, A\&A, 423, 849

[8] Lin, C. C., \& Shu, F. H. 1964, APJ, 140, 646

[9] Mcmillan, P. J. 2011, MNRAS, 414, 2446

[10] Miller, R. H., Prendergast, K. H., \& Quirk, W. J. 1970, ApJ, 161, 903

[11] Piñol-Ferrer, N., Lindblad, P. O., \& Fathi, K. 2012, MNRAS, 421, 1089

[12] Reid, M. J., Menten, K. M., Zheng, X. W., et al. 2009, ApJ, 700, 137

[13] Reid, M. J., \& Honma, M. 2014a, ARA\&A, 52, 339

[14] Reid, M. J., Menten, K. M., Brunthaler, A., et al. 2014b, ApJ, 783, 130

[15] Roberts, W. W. 1969, APJ, 158, 123

[16] Sakai, N., Nakanishi, H., Matsuo, M., et al. 2015 in submitted, PASJ

[17] Vallée., J. P. 2014, AJ, 148, 5 


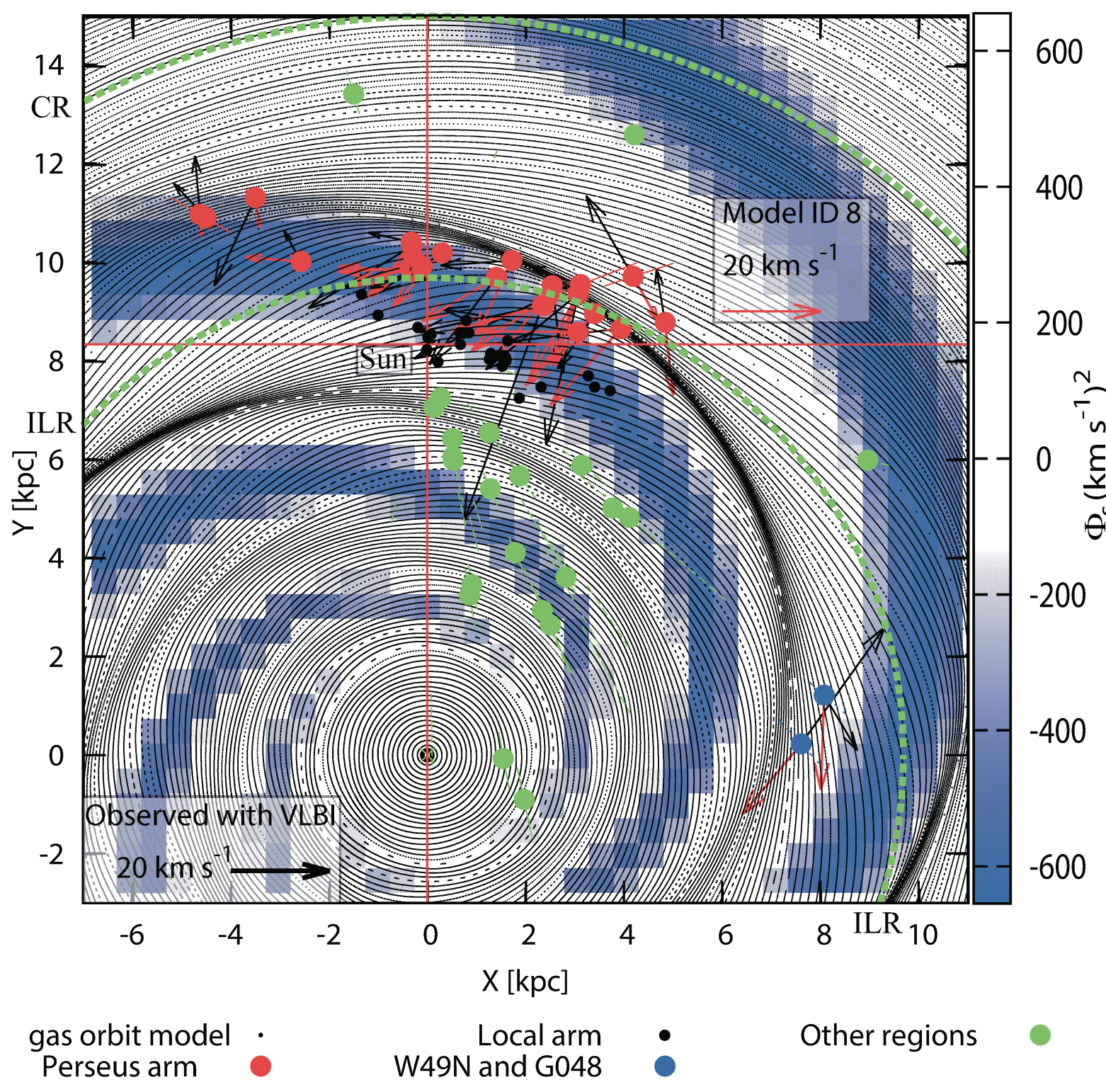

Figure 1: The gas orbits model generated from the model ID-8 listed in table 1 (Sakai et al. 2015 in submitted). Circles show previous VLBI astrometry results, and different colors mean different regions (e.g., Red = Perseus arm; Black = Local arm; Blue $=\mathrm{W} 49 \mathrm{~N}$ and G48.60+0.02; Green = others). Black and red arrows show observed and modeled peculiar (non-circular) motions, respectively. Note that we assumed a flat rotation model (i.e., $\left.\Theta(R)=\Theta_{0}\right)$ and Galactic constants of $\left(R_{0}, \Theta_{0}\right)=(8.33$ $\mathrm{kpc}, 240.0 \mathrm{~km} \mathrm{~s}^{-1}$ ) to derive the peculiar motions. Here $\Theta(R)$ shows the rotation speed at a Galactocentric distance $R$. Color bar represents the amplitude of the spiral potential model (ID-8). Inner and outer dotted green curves show the Inner Lindblad Resonance (ILR) and the Corotation Resonance (CR), respectively. 